\title{
Resonance asymptotics for a pair quantum waveguides with common semitransparent perforated wall
}

\author{
A. M. Vorobiev, E. S. Trifanova, I. Y. Popov \\ ITMO University, Kronverkskiy, 49, St. Petersburg, 197101, Russia \\ lenden31@yandex.ru, etrifanova@gmail.com,popov1955@gmail.com
}

DOI 10.17586/2220-8054-2020-11-6-619-627

\begin{abstract}
A nanostructure model,which is represented as a pair of coupled two-dimensional quantum waveguides with common semitransparent wall, is considered. That wall has small window which induces a resonance state localized near the window. Semitransparency is the reason for the asymptotics difference in comparison with the non-transparent case. Using the matching of asymptotic expansions method, we obtain formulas for resonances and resonance states.
\end{abstract}

Keywords: resonance, resonance state, waveguide, asymptotics.

Received: 29 November 2020

\section{Introduction}

There is a large class of mathematical and physical problems concerning "systems with small coupling windows", actually this means systems of connected quantum waveguides, Helmholtz resonators, and other structures with perturbation caused by small coupling windows. Such systems have long been of interest to physicists and mathematicians. The transport properties of waveguides and other additional phenomena caused by resonators are widely used in electrodynamics, radiophysics and theories of acoustic and electromagnetic waves.

The development of nanoelectronics led to the creation of a new class of objects used inside devices - quantum threads (nanowhiskers), quantum dots, antidots and so on. Of course, "quantum" is the keyword here because studying mesoscopic systems (systems where the coherence of the phases of the electrons is preserved on a scale much larger than atomic) is absolutely impossible if we don't respect quantum properties of electron. Obviously, taking into account the quantum behavior of an electron requires the development of fundamentally new physical, and, most importantly, also mathematical approaches.

When we consider mesoscopic systems, we actually mean studying wave propagation in waveguides and other structures. Properties of this propagation are related to spectral properties of the corresponding mathematical operator. This is usually the Schrödinger operator which coincides with the Laplace operator for the ballistic regime. Resonance phenomena are widely used for development of new nanoelectronic devices. Knowledge about the quasi-eigenvalue asymptotics allows one to ensure a proper control of the electron transmission through the device.

Such problems have a long history. They became interesting for mathematicians and theoretical physics after Rayleigh's work in 1916 [1]. Probably he was the first who successfully considered the Helmholtz resonator from a mathematical point of view. He calculated the real part of the smallest quasi-eigenvalue. Although it was not very significant physical result, that work used a real mathematical approach so it was the beginning of the story. Rayleigh's result was not significant, because the imaginary part of quasi-eigenvalue is much more important - it actually represents time of resonance states existing and it is really important physical metric. Half a century later imaginary part was calculated by Morse and Feshbach [2] using the Rayleigh's method.

Such problems are popular today as well, as they became after first mathematical approaches. Currently, we use term "asymptotic analysis" for big class of problem including resonance phenomena. Great variety of electronic device caused great variety of systems in resonance problems. Firstly, a system can contain different units like quantum waveguides, Helmholtz resonators, angles and so on. Also it can contain combination of such units. There are several examples, such as two connected resonators [3], a single resonator opened to space [4], a pair of waveguides with A common boundary [5] or even A system of waveguides with resonators [6]. System geometry is also changeable factor, so we can vary shape of resonator (like square, circle, arbitrary domain) [7], number of dimensions (plane is not less actual than three-dimensional case) [8], window position and numbers (both are explicit physical factors, but they can lead us to quite different results) $[9,10]$. Changing of the boundary conditions in the problem was studied by Gadylshin and Borisov [11].

The core of mathematical approach in this work is "Matching asymptotic expansions of solutions of boundary problems". This method was described in prior literature [12]. Let's consider a global problem, the solution of which necessitates a similar tool. This method is intended for boundary problems of equations containing naturally occurring 
small parameter. This is very typical for mathematical physics problems, in particular for problems with Helmholtz resonator and quantum waveguides where the small parameter characterizes the window size. The most interesting problem is that solution in such problems almost alway can't be decomposed into some simple functions. For example, in the Helmholtz resonator with the small perturbation we can't express the above exact decision so we only can use asymptotic expansions.

We will consider a couple of waveguides in which the common wall is semitransparent. Such a system was studied by Exner and Kreicirik in $[13,14]$ but in terms of spectral and scattering properties. Resonance asymptotics for a single waveguide with semitransparent barrier were considered in [15]. Semitransparency is also related to previous paragraph because, in our case, specific boundary conditions on the common wall are required.

\section{Preliminaries}

Let us describe the system and prepare common formulas, which are required to construct our case-specific asymptotics.

There is a pair of quantum waveguides in two-dimensional Cartesian coordinates with widths $d_{-}$and $d_{+}$. We will consider $d_{-}<d_{+}<2 d_{-}$, which is a very important assumption, as we will see later.

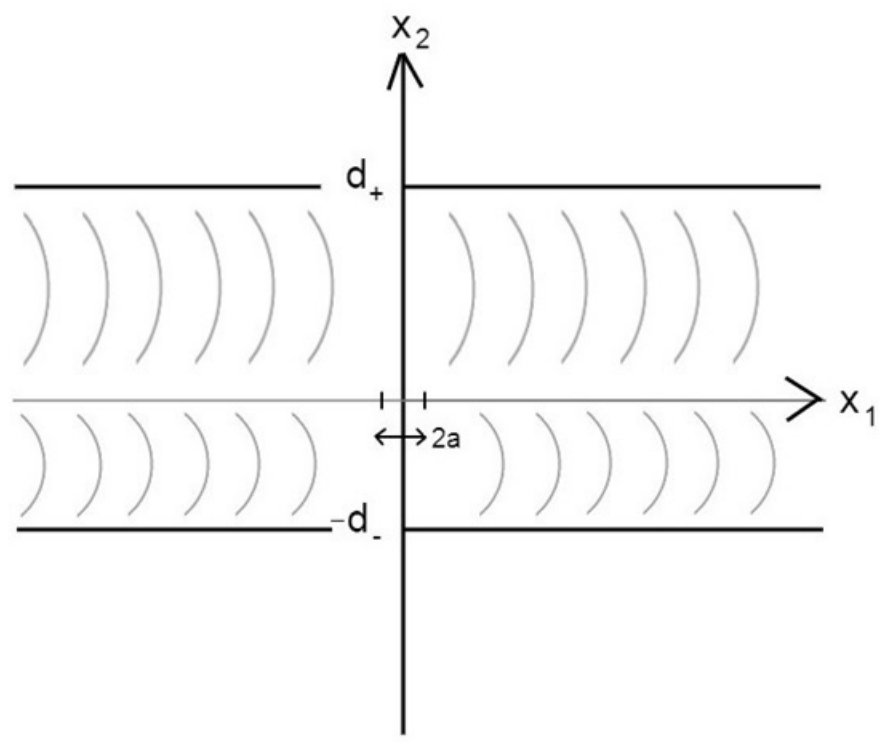

FIG. 1. waveguides with common semitransparent wall

Wall semitransparency is described by the parameter $\alpha$. Generally $\alpha \in(0 ;+\infty)$, where zero value means no barrier and infinity means absolutely nontransparent barrier. Zero value is not included because actually it is not a case of considered problem.

As mentioned previously, boundary conditions on the walls are very important. On non-common walls, we choose the Dirichlet conditions, but common wall conditions are more complicated. When a wave passes through the barrier, a jump occurs in the derivative of the considered function $u\left(x_{1}, x_{2}\right)$, so we will set specific boundary conditions:

$$
\left\{\begin{array}{l}
u_{+}=u_{-}, \\
u_{+}^{\prime}-u_{-}^{\prime}=\alpha u,
\end{array}\right.
$$

where $u_{+-}^{\prime}$ are vertical derivatives at the top and bottom of the wall. The conditions of such type appear if one considers singular potential supported on hypersurface. These potentials are intensively investigated during last two decades (see, e.g., [16-22]).

We construct asymptotics in window size, so one of the most important parameters is $a$ - we will consider it as half of the window size.

As mentioned previously, the most important point is selected threshold. In similar problems, resonances are considered near "threshold" values. Threshold is such value of $\lambda_{n}$, that there are no summands with imaginary part exponent in Green's function series which are lower than $\lambda_{n}$. Imaginary exponent corresponds to periodic summands 
which mean propagating waves. We will seek terms of asymptotic expansions close to the first threshold $\lambda_{1}^{-}=$ $\left(\frac{\pi}{d_{-}}\right)^{2}$. Choice of $\left(\frac{\pi}{d_{-}}\right)^{2}$ but not $\left(\frac{\pi}{d_{+}}\right)^{2}$ is also key point as we will see later.

"Perturbed" eigenvalue $k_{a}^{2}$. also known as quasi-eigenfrequency will be denoted as $k_{a}$. The difference between $k_{a}^{2}$ and $\lambda_{1}^{-}$is actually small value. We will use convenient expansion for its asymptotics:

$$
\sqrt{\left(\frac{\pi}{d_{-}}\right)^{2}-k_{a}^{2}}=\sum_{j=2}^{\infty} \sum_{i=0}^{[j / 2]-1} k_{j i} a^{j} \ln ^{i} \frac{a}{a_{0}} .
$$

Finally, we can write down system of equations for eigenfunctions in perturbed case:

$$
\left\{\begin{array}{l}
\psi_{a}(x)=-\left.\sqrt{\left(\frac{\pi}{d_{-}}\right)^{2}-k_{a}^{2}} \cdot \sum_{j=0}^{\infty} a^{j} P_{j+1}\left(D_{y}, \ln \frac{a}{a_{0}}\right) G^{+}(x, y, k)\right|_{y=0}, \quad x \in \Omega^{+} \backslash S_{a_{0}\left(a / a_{0}\right)^{1 / 2}}, \\
\psi_{a}(x)=\sum_{j=1}^{\infty} \sum_{i=0}^{[(j-1) / 2]} v_{j i}\left(\frac{x}{a}\right) a^{j} \ln ^{i} \frac{a}{a_{0}}, \quad x \in S_{2 a_{0}\left(a / a_{0}\right)^{1 / 2},} \\
\psi_{a}(x)=\left.\sqrt{\left(\frac{\pi}{d_{-}}\right)^{2}-k_{a}^{2}} \cdot \sum_{j=0}^{\infty} a^{j} P_{j+1}\left(D_{y}, \ln \frac{a}{a_{0}}\right) G^{-}(x, y, k)\right|_{y=0}, \quad x \in \Omega^{-} \backslash S_{a_{0}\left(a / a_{0}\right)^{1 / 2}} .
\end{array}\right.
$$

\section{Asymptotics construction}

\subsection{Eigenfunctions for semitransparent wall}

Eigenfunctions and corresponding eigenvalues for case without semitransparency and perturbing are well-known:

$$
\left\{\begin{array}{l}
\psi_{n}^{ \pm}(x)=\sqrt{\frac{2}{d_{ \pm}}} \sin \frac{\pi n x_{2}}{d_{ \pm}}, \\
\lambda_{n}^{ \pm}=\left(\frac{\pi n}{d_{ \pm}}\right)^{2} .
\end{array}\right.
$$

But we should match conditions (1), so let's consider eigenfunction in such form:

$$
\chi_{n}(x)= \begin{cases}A_{n} \sin \left(\left(x_{2}-d_{+}\right) \nu\right), & x_{2}>0, \\ B_{n} \sin \left(\left(x_{2}+d_{-}\right) \nu\right), & x_{2}<0 .\end{cases}
$$

So we obtain then following equations:

$$
\begin{aligned}
& \left\{\begin{array}{l}
-A_{n} \sin \left(d_{+} \nu\right)=B_{n} \sin \left(d_{-} \nu\right), \\
A_{n} \nu \cos \left(d_{+} \nu\right)-B_{n} \nu \cos \left(d_{-} \nu\right)=\alpha B_{n} \sin \left(d_{-} \nu\right) .
\end{array} \Leftrightarrow\right. \\
& \left\{\begin{array}{l}
\frac{A_{n}}{B_{n}}=-\frac{\sin \left(d_{-} \nu\right)}{\sin \left(d_{+} \nu\right)}, \\
-B_{n} \frac{\sin \left(d_{-} \nu\right)}{\sin \left(d_{+} \nu\right)} \nu \cos \left(d_{+} \nu\right)-B_{n} \nu \cos \left(d_{-} \nu\right)=\alpha B_{n} \sin \left(d_{-} \nu\right) .
\end{array} \Leftrightarrow\right. \\
& \left\{\begin{array}{l}
\frac{A_{n}}{B_{n}}=-\frac{\sin \left(d_{-} \nu\right)}{\sin \left(d_{+} \nu\right)} \\
-\nu \operatorname{ctg}\left(d_{+} \nu\right)-\nu \operatorname{ctg}\left(d_{-} \nu\right)=\alpha .
\end{array}\right. \\
& \chi_{n}(x)=\left\{\begin{array}{l}
-C_{n} \sin \left(d_{-} \nu\right) \sin \left(\left(x_{2}-d_{+}\right) \nu\right), \quad x_{2}>0, \\
C_{n} \sin \left(d_{+} \nu\right) \sin \left(\left(x_{2}+d_{-}\right) \nu\right), \quad x_{2}<0 .
\end{array}\right.
\end{aligned}
$$

$C_{n}$ is normalizing coefficient. Actually it means that $\nu$ can be found as solution of equation

$$
-\nu \cot \left(d_{+} \nu\right)-\nu \cot \left(d_{-} \nu\right)=\alpha
$$

but we won't consider corresponding formulas in details. 


\subsection{Green's function}

Matching of asymptotic expansions corresponding to (3) requires Green's function calculating. Its common formula for a waveguide is well known [23]:

$$
G^{ \pm}(x, y, k)=\sum_{n=1}^{\infty} \frac{\chi_{n}\left(x_{2}\right) \cdot \chi_{n}\left(y_{2}\right)}{2 p_{n}^{ \pm}} \cdot e^{-p_{n}^{ \pm}\left(x_{1}-y_{1}\right)},
$$

where $p_{n}^{ \pm}=\sqrt{\left(\frac{\pi n}{d_{ \pm}}\right)^{2}-k_{a}^{2}}$.

For our expressions, it takes the form:

$$
\left\{\begin{array}{l}
G^{+}(x, y, k)=\sum_{n=1}^{\infty} \frac{C_{n}^{2} \sin ^{2}\left(d_{-} \nu\right) \sin \left(\left(x_{2}-d_{+}\right) \nu\right) \sin \left(\left(y_{2}-d_{+}\right) \nu\right)}{2 p_{n}^{+}} \cdot e^{-p_{n}^{+}\left(x_{1}-y_{1}\right)}, \\
G^{-}(x, y, k)=\sum_{n=1}^{\infty} \frac{C_{n}^{2} \sin ^{2}\left(d_{+} \nu\right) \sin \left(\left(x_{2}+d_{-}\right) \nu\right) \sin \left(\left(y_{2}+d_{-}\right) \nu\right)}{2 p_{n}^{-}} \cdot e^{-p_{n}^{-}\left(x_{1}-y_{1}\right)} .
\end{array}\right.
$$

The differential operator $P_{n}$ from formula (3) can be described as follows:

$$
\begin{gathered}
P_{0}\left(D_{y}, \ln \frac{\varepsilon}{\varepsilon_{0}}\right)=a_{10}^{(0)} I, \quad P_{1}\left(D_{y}, \ln \frac{\varepsilon}{\varepsilon_{0}}\right)=a_{10}^{(1)} D_{y}^{1}, \quad D_{y}^{n}=\frac{\partial^{n}}{\partial n_{y}^{n}}, \\
P_{m}\left(D_{y}, \ln \frac{\varepsilon}{\varepsilon_{0}}\right)=\sum_{q=1}^{m-1} \sum_{i=0}^{[(q-1) / 2]} a_{q i}^{(m)}\left(\ln \frac{\varepsilon}{\varepsilon_{0}}\right)^{i} D_{y}^{m-q+1}, \quad m \geq 2 .
\end{gathered}
$$

Actually, operator $D_{y}$ should be just a combination of $\mathrm{n}$ tangent or normal derivatives in dot y. Here, we choose always normal derivatives. Finally, we obtain the following representation for $D_{y}^{n} G$ :

$$
\begin{aligned}
& D_{y}^{j} G^{ \pm}(x, 0, k)=\left.\frac{C_{1}^{2} \sin ^{2}\left(d_{\mp} \nu\right)\left(\sin \left(x_{2} \nu\right) \cos \left(d_{ \pm} \nu\right) \mp \cos \left(x_{2} \nu\right) \sin \left(d_{ \pm} \nu\right)\right) D_{y}^{j} \sin \left(\left(y_{2} \mp d_{ \pm}\right) \nu\right)}{2 p_{1}^{ \pm}}\right|_{y=0} \cdot e^{-x_{1} p_{1}^{ \pm}} \\
& +\Phi_{j}(x, k) \ln \frac{r}{a_{0}}+g_{j}^{ \pm}(x, k)+\sum_{i=0}^{[j / 2]} \sum_{t=0}^{j-2 i-1} b_{i t}^{(j)} r^{-j+2(i+t)}\left(\cos (j-2 i) \theta \pm \frac{\alpha}{2(j-2 i)} \sin (j-2 i) \theta\right) .
\end{aligned}
$$

where $(r, \theta)$ are polar coordinates. Terms $b_{i t}^{(j)}, \Phi_{j}(x, k), g_{j}^{ \pm}(x, k)$ are analytic in respect to $\tau$ some vicinity of the point $\lambda_{1}$ :

$$
b_{00}^{j}=(-1)^{[(j+1) / 2]}(j-1) ! / \pi, \quad b_{10}^{3}=\frac{k^{2}}{2 \pi}=\frac{\pi}{2 d_{-}^{2}}, \quad \Phi_{1 n}(0, k)=-\frac{\tau^{2}}{2 \pi}=-\frac{\pi}{2 d_{-}^{2}} .
$$

Sines and cosines in last summand are selected in such way because $D_{y}^{n} G$ should satisfy conditions (1).

Let's notice that the first summand has imaginary part and no k-singularity for $G^{+}$and has no imaginary part but k-singularity for $G^{-}$.

\subsection{Calculating}

Boundary problems for $v_{j i}\left(\frac{x}{a}\right)$ from (3) can be obtained by the following manner. We substitute the series (3) and (2) into the Helmholtz equation (for $k=k_{a}$ ) and then change variables $\xi=\frac{x}{a}$. The coefficients in the terms with the same powers of $a$ and $\ln \frac{a}{a_{0}}$ should be equal. Hence, we obtain the following problems:

$$
\begin{aligned}
& \Delta_{\xi} v_{j i}=-\sum_{p=0}^{j-3} \sum_{q=0}^{[p / 2]} \Lambda_{p q} v_{j-p-2, i-q}, \quad \xi \in R^{2} \backslash \gamma \\
& v_{j i}=0, \quad \xi \in \gamma
\end{aligned}
$$

where $\gamma=\left\{\xi \mid \xi_{2}=0 \wedge \xi_{1} \in(-\infty ;-1] \cup[1 ;+\infty)\right\}$ and $\Lambda_{p q}$ are the coefficients of the series:

$$
k_{a}^{2}=\sum_{p} \sum_{q} \Lambda_{p q} a^{p} \ln ^{q} \frac{a}{a_{0}} .
$$

We can notice for future that (6) gives us the homogeneous Laplace equation for $v_{10}$ and $v_{20}$ but for $v_{30}$ we get the inhomogeneous Laplace equation (Poisson equation) and it looks like $\Delta_{\xi} v_{30}=-k_{0}^{2} v_{10}$ because of corresponding $\Lambda_{00}$ value. 
As a next step, we need to introduce operator $M_{p q}(U)$ - it changes variables in expressions $\mathrm{U}\left(\xi=\frac{x}{\varepsilon}, \ln r=\right.$ $\ln \rho+\ln \varepsilon)$ and filters summand with $\varepsilon^{p} \ln ^{q} \frac{\varepsilon}{\varepsilon_{0}} \varphi(\xi)$. Also $M_{p}=\sum_{q} M_{p q}$, it is used to get all summand with $\varepsilon^{p}$.

\section{Calculating of $\mathbf{k}_{\mathbf{2 0}}$}

Let's find summand of order $a^{1}$ from (3) using this operator.

$$
\begin{gathered}
a^{-1} M_{1}\left(-\sqrt{\frac{\pi^{2}}{d_{-}^{2}}-k_{a}^{2}} \cdot P_{1} G^{+}\left(x, 0, k_{a}\right)\right)=\frac{1}{\pi} k_{20} a_{10}^{(1)} \rho^{-1}\left(\cos \theta+\frac{\alpha}{2} \sin \theta\right) \\
a^{-1} M_{1}\left(\sqrt{\frac{\pi^{2}}{d_{-}^{2}}-k_{a}^{2}} \cdot P_{1} G^{-}\left(x, 0, k_{a}\right)\right)=-\frac{1}{\pi} k_{20} a_{10}^{(1)} \rho^{-1}\left(\cos \theta-\frac{\alpha}{2} \sin \theta\right)+\rho \sin \theta \cdot \frac{a_{10}^{(1)} C_{1}^{2} \sin ^{2}\left(d_{+} \nu\right) \nu^{2} \cos ^{2}\left(d_{-} \nu\right)}{2} .
\end{gathered}
$$

To find $v_{10}$, we use the following lemma:

There exist harmonic functions $Y_{q 1}(\xi), Y_{q 2}(\xi)$ in $\mathbb{R}^{2} \backslash(\mathbb{R} \backslash(-1 ; 1)),\left.Y_{q s}\right|_{\mathbb{R} \backslash(-1 ; 1)}=0, Y_{q s} \in W_{2, l o c}^{1}\left(\mathbb{R}^{2}\right)$, which have the following differentiable asymptotics by $\rho, \rho \rightarrow \infty$ :

$$
\begin{aligned}
& Y_{q 1}=\left\{\begin{array}{l}
-\sum_{j=1}^{\infty} \rho^{-j} a_{q j}^{+} \cos j \theta, \quad \xi_{2}>0, \\
\rho^{q} a_{q}^{0} \cos q \theta+\sum_{j=1}^{\infty} \rho^{-j} a_{q j}^{-} \cos j \theta, \quad \xi_{2}<0,
\end{array}\right. \\
& Y_{q 2}=\left\{\begin{array}{l}
-\sum_{j=1}^{\infty} \rho^{-j} b_{q j}^{+} \sin j \theta, \quad \xi_{2}>0, \\
\rho^{q} b_{q}^{0} \sin q \theta+\sum_{j=1}^{\infty} \rho^{-j} b_{q j}^{-} \sin j \theta, \quad \xi_{2}<0 .
\end{array}\right.
\end{aligned}
$$

Each harmonic in $\mathbb{R}^{2} \backslash(\mathbb{R} \backslash(-1 ; 1))$ function $\mathrm{V}$ that is 0 on $\mathbb{R} \backslash(-1 ; 1)$ and has the order $O\left(\rho^{q}\right)$ is a linear combination of $Y_{j 1}(\xi), Y_{j 2}(\xi), Y_{j 1}(\xi *), Y_{j 2}(\xi *)$ for $j \leq q$, where $\xi *=\left(\xi_{1},-\xi_{2}\right)$.

To match terms increasing on $\rho \rightarrow \infty$ in according to (8) we shall select $v_{10}(\xi)$ in such way:

$$
v_{10}(\xi)=\frac{a_{10}^{(1)} C_{1}^{2} \sin ^{2}\left(d_{+} \nu\right) \nu^{2} \cos ^{2}\left(d_{-} \nu\right)}{2 b_{1}^{0}} Y_{12}(\xi) .
$$

Hence, matching terms of order $\rho^{-1} \sin \theta$ in (7), (8) with $Y$ can be used as follows:

And finally:

$$
\left\{\begin{array}{l}
\frac{a_{10}^{(1)} C_{1}^{2} \sin ^{2}\left(d_{+} \nu\right) \nu^{2} \cos ^{2}\left(d_{-} \nu\right) b_{11}^{-}}{2 b_{1}^{0}}=\frac{\alpha}{2 \pi} k_{20} a_{10}^{(1)} \\
b_{11}^{-}=-b_{11}^{+} .
\end{array}\right.
$$

$$
k_{20}=\frac{\pi C_{1}^{2} \sin ^{2}\left(d_{+} \nu\right) \nu^{2} \cos ^{2}\left(d_{-} \nu\right) b_{11}^{-}}{b_{1}^{0} \alpha}
$$

\section{Calculating of $\mathbf{k}_{\mathbf{3 0}}$}

Process of calculating for next coefficient is not much different.

Positive powers of $\rho$ appear from summands of order $a^{2}$ in $P_{1}, P_{2}$, so:

$$
\begin{aligned}
& a^{-2} M_{2}\left(-\sqrt{\frac{\pi^{2}}{d_{-}^{2}}-k_{a}^{2}} \cdot P_{1} G^{+}\left(x, 0, k_{a}\right)\right)=\frac{1}{\pi} k_{30} a_{10}^{(1)} \rho^{-1}\left(\cos \theta+\frac{\alpha}{2} \sin \theta\right), \\
& a^{-2} M_{2}\left(\sqrt{\frac{\pi^{2}}{d_{-}^{2}}-k_{a}^{2}} \cdot P_{1} G^{-}\left(x, 0, k_{a}\right)\right)=-\frac{1}{\pi} k_{30} a_{10}^{(1)} \rho^{-1}\left(\cos \theta-\frac{\alpha}{2} \sin \theta\right)+ \\
& +\rho^{2} \cos 2 \theta \cdot \frac{a_{10}^{(1)} C_{1}^{2} \sin ^{2}\left(d_{+} \nu\right) \nu^{3} \cos \left(d_{-} \nu\right) \sin \left(d_{-} \nu\right)}{8}, \\
& a^{-2} M_{2}\left(-\sqrt{\frac{\pi^{2}}{d_{-}^{2}}-k_{a}^{2}} \cdot a \cdot P_{2} G^{+}\left(x, 0, k_{a}\right)\right)=\frac{1}{\pi} k_{30} a_{10}^{(2)} \rho^{-2}\left(\cos 2 \theta+\frac{\alpha}{4} \sin 2 \theta\right),
\end{aligned}
$$




$$
\begin{aligned}
& a^{-2} M_{2}\left(\sqrt{\frac{\pi^{2}}{d_{-}^{2}}-k_{a}^{2}} \cdot a \cdot P_{2} G^{-}\left(x, 0, k_{a}\right)\right)=-\frac{1}{\pi} k_{30} a_{10}^{(2)} \rho^{-2}\left(\cos 2 \theta-\frac{\alpha}{4} \sin 2 \theta\right)+ \\
& +\rho \sin \theta \cdot \frac{-a_{10}^{(2)} C_{1}^{2} \sin ^{2}\left(d_{+} \nu\right) \nu^{3} \cos \left(d_{-} \nu\right) \sin \left(d_{-} \nu\right)}{2} .
\end{aligned}
$$

In (9)-(12) we consider only summands with $\operatorname{deg} \rho \in\{-1\} \cup N$, actually right side in (11) is not 0 of course.

Because of given positive degrees of $\rho$ we shall select $v_{20}(\xi)$ in such way:

$$
v_{20}(\xi)=-\frac{a_{10}^{(2)} C_{1}^{2} \sin ^{2}\left(d_{+} \nu\right) \nu^{3} \cos \left(d_{-} \nu\right) \sin \left(d_{-} \nu\right)}{2 b_{1}^{0}} Y_{12}(\xi)+\frac{a_{10}^{(1)} C_{1}^{2} \sin ^{2}\left(d_{+} \nu\right) \nu^{3} \cos \left(d_{-} \nu\right) \sin \left(d_{-} \nu\right)}{8 a_{2}^{0}} Y_{21}(\xi) .
$$

Hence matching terms of order $\rho^{-1} \sin \theta$ and $\rho^{-1} \cos \theta$ in (7), (8) with $Y$ can be used as follows:

$$
\left\{\begin{array}{l}
\frac{-a_{10}^{(2)} C_{1}^{2} \sin ^{2}\left(d_{+} \nu\right) \nu^{3} \cos \left(d_{-} \nu\right) \sin \left(d_{-} \nu\right)}{2 b_{1}^{0}}\left(-a_{21}^{+}\right)=\frac{1}{\pi} k_{30} a_{10}^{(1)} \\
\frac{-a_{10}^{(2)} C_{1}^{2} \sin ^{2}\left(d_{+} \nu\right) \nu^{3} \cos \left(d_{-} \nu\right) \sin \left(d_{-} \nu\right)}{2 b_{1}^{0}}\left(-a_{22}^{+}\right)=\frac{1}{\pi} k_{30} a_{10}^{(2)} \\
\frac{a_{10}^{(1)} C_{1}^{2} \sin ^{2}\left(d_{+} \nu\right) \nu^{3} \cos \left(d_{-} \nu\right) \sin \left(d_{-} \nu\right)}{8 a_{2}^{0}}\left(-b_{11}^{+}\right)=-\frac{\alpha}{2 \pi} k_{30} a_{10}^{(1)} \\
\frac{a_{10}^{(1)} C_{1}^{2} \sin ^{2}\left(d_{+} \nu\right) \nu^{3} \cos \left(d_{-} \nu\right) \sin \left(d_{-} \nu\right)}{8 a_{2}^{0}}\left(-b_{12}^{+}\right)=-\frac{\alpha}{4 \pi} k_{30} a_{10}^{(2)}, \\
a_{21}^{+}=a_{21}^{-}, \\
a_{22}^{+}=a_{22}^{-} \\
b_{12}^{+}=-b_{12}^{-}
\end{array}\right.
$$

Finally:

$$
k_{30}=\frac{\pi a_{22}^{+} C_{1}^{2} \sin ^{2}\left(d_{+} \nu\right) \nu^{3} \cos \left(d_{-} \nu\right) \sin \left(d_{-} \nu\right)}{2 b_{1}^{0}} .
$$

Using other equations we can obtain relations for coefficients.

\section{Calculating of $\mathbf{k}_{40}, \mathbf{k}_{41}$}

As we noticed previously, equation (6) is homogeneous for $v_{10}(\xi), v_{20}(\xi)$ but becomes more complicated for next step. So we need to solve Poisson equation: $\Delta_{\xi} v_{30}=-\frac{\pi^{2}}{d_{-}^{2}} v_{10}, v_{30}(\xi)=0, \xi \in \gamma, \gamma=\left\{\xi \mid \xi_{2}=0 \wedge \xi_{1} \in\right.$ $(-\infty ;-1] \cup[1 ;+\infty)\}$.

Solution of this boundary problem can be presented as:

$$
v_{30}(\xi)=\widehat{v}_{30}(\xi)+\widetilde{v}_{30}(\xi),
$$

where $\widehat{v}_{30}(\xi)$ is solution of homogeneous Laplace equation satisfying the boundary conditions (as we seek for previous steps) and $\widetilde{v}_{30}(\xi)$ is particular solution of inhomogeneous Laplace equation satisfying the boundary conditions.

Let's separate summands of order $a^{3}$. To get all positive degrees of $\rho$ it's enough to consider $P_{1}, P_{2}, P_{3}$. Analogously to (9)-(12), we won't get profit (except coefficients relations) considering any negative degrees except -1 , so we will consider only -1 from negative $\rho$ degrees:

$$
\begin{aligned}
& a^{-3} M_{30}\left(-\sqrt{\frac{\pi^{2}}{d_{-}^{2}}-k_{a}^{2}} \cdot P_{1} G^{+}\left(x, 0, k_{a}\right)\right)=\rho^{-1} \frac{1}{\pi}\left(\cos \theta+\frac{\alpha}{2} \sin \theta\right) \cdot a_{10}^{(1)} k_{40}+ \\
& +\rho \sin \theta \cdot\left[-a_{10}^{(1)} k_{20} g_{x}^{+}+i \frac{C_{1}^{2} \sin ^{2}\left(d_{-} \nu\right) a_{10}^{(1)} k_{20} \nu^{2} d_{-} d_{+} \cos ^{2}\left(d_{+} \nu\right)}{2 \pi \sqrt{d_{+}^{2}-d_{-}^{2}}}\right]- \\
& -\rho \cos \theta \frac{C_{1}^{2} \sin ^{2}\left(d_{-} \nu\right) a_{10}^{(1)} k_{20} \nu \cos \left(d_{+} \nu\right) \sin \left(d_{+} \nu\right)}{2}
\end{aligned}
$$




$$
\begin{aligned}
& a^{-3} M_{30}\left(\sqrt{\frac{\pi^{2}}{d_{-}^{2}}-k_{a}^{2}} \cdot P_{1} G^{-}\left(x, 0, k_{a}\right)\right)=-\rho^{-1} \frac{1}{\pi}\left(\cos \theta-\frac{\alpha}{2} \sin \theta\right) a_{10}^{(1)} k_{40}+ \\
& +\rho \sin \theta \cdot a_{10}^{(1)} k_{20} g_{-}^{+}-\rho \cos \theta \frac{C_{1}^{2} \sin ^{2}\left(d_{+} \nu\right) a_{10}^{(1)} k_{20} \nu \cos \left(d_{-} \nu\right) \sin \left(d_{-} \nu\right)}{2}+ \\
& +\rho^{3} \sin 3 \theta \frac{C_{1}^{2} \sin ^{2}\left(d_{+} \nu\right) a_{10}^{(1)} \nu^{4} \cos ^{2}\left(d_{-} \nu\right)}{48} \\
& a^{-3} M_{30}\left(-\sqrt{\frac{\pi^{2}}{d_{-}^{2}}-k_{a}^{2}} \cdot a \cdot P_{2} G^{+}\left(x, 0, k_{a}\right)\right)=0, \\
& a^{-3} M_{30}\left(\sqrt{\frac{\pi^{2}}{d_{-}^{2}}-k_{a}^{2}} \cdot a \cdot P_{2} G^{-}\left(x, 0, k_{a}\right)\right)=-\rho^{2} \cos 2 \theta \frac{C_{1}^{2} \sin ^{2}\left(d_{+} \nu\right) a_{10}^{(2)} \nu^{4} \sin ^{2}\left(d_{-} \nu\right)}{8}, \\
& a^{-3} M_{30}\left(-\sqrt{\frac{\pi^{2}}{d_{-}^{2}}-k_{a}^{2}} \cdot a^{2} \cdot P_{3} G^{+}\left(x, 0, k_{a}\right)\right)=-\rho^{-1}\left(\cos \theta+\frac{\alpha}{2} \sin \theta\right) \cdot\left[a_{10}^{(3)} b_{10}^{(3)} k_{20}+3 a_{10}^{(3)} b_{01}^{(3)} k_{20}\right], \\
& a^{-3} M_{30}\left(\sqrt{\frac{\pi^{2}}{d_{-}^{2}}-k_{a}^{2}} \cdot a^{2} \cdot P_{3} G^{-}\left(x, 0, k_{a}\right)\right)= \\
& \rho \sin \theta \cdot\left[-\frac{C_{1}^{2} \sin ^{2}\left(d_{+} \nu\right) a_{10}^{(3)} k_{20} \nu^{4} \cos ^{2}\left(d_{-} \nu\right)}{2}-\frac{C_{1}^{2} \sin ^{2}\left(d_{+} \nu\right) a_{20}^{(3)} k_{20} \nu^{3} \cos \left(d_{-} \nu\right) \sin \left(d_{-} \nu\right)}{2}\right]+ \\
& +\rho^{-1}\left(\cos \theta+\frac{\alpha}{2} \sin \theta\right) \cdot\left[a_{10}^{(3)} b_{10}^{(3)} k_{20}+3 a_{10}^{(3)} b_{01}^{(3)} k_{20}\right] .
\end{aligned}
$$

Separating summands with positive $\rho$ degrees we can obtain the following representation for $\widehat{v}_{30}(\xi)$ :

$$
\widehat{v}_{30}(\xi)=\beta_{11} Y_{11}(\xi)+\beta_{12} Y_{12}(\xi)+\beta_{21} Y_{21}(\xi)+\beta_{32} Y_{32}(\xi)+\widetilde{\beta}_{11} Y_{11}(\xi *)+\widetilde{\beta}_{12} Y_{12}(\xi *)
$$

where:

$$
\begin{aligned}
& \beta_{11}=-\frac{C_{1}^{2} \sin ^{2}\left(d_{+} \nu\right) a_{10}^{(1)} k_{20} \nu \sin \left(d_{-} \nu\right) \cos \left(d_{-} \nu\right)}{2 a_{1}^{0}}, \\
& \beta_{12}=\frac{a_{10}^{(1)} k_{20} g_{x}^{-}}{b_{1}^{0}}-\frac{C_{1}^{2} \sin ^{2}\left(d_{+} \nu\right) a_{10}^{(3)} \nu^{4} \cos ^{2}\left(d_{-} \nu\right)}{2 b_{1}^{0}}-\frac{C_{1}^{2} \sin ^{2}\left(d_{+} \nu\right) a_{20}^{(3)} \nu^{3} \sin \left(d_{-} \nu\right) \cos \left(d_{-} \nu\right)}{2 b_{1}^{0}}, \\
& \beta_{21}=-\frac{C_{1}^{2} \sin ^{2}\left(d_{+} \nu\right) a_{10}^{(2)} \nu^{4} \sin ^{2}\left(d_{-} \nu\right)}{8 a_{2}^{0}}, \\
& \beta_{32}=\frac{C_{1}^{2} \sin ^{2}\left(d_{+} \nu\right) a_{10}^{(1)} \nu^{4} \cos ^{2}\left(d_{-} \nu\right)}{48 b_{3}^{0}}, \\
& \widetilde{\beta}_{11}=-\frac{C_{1}^{2} \sin ^{2}\left(d_{-} \nu\right) a_{10}^{(1)} k_{20} \nu \sin \left(d_{+} \nu\right) \cos \left(d_{+} \nu\right)}{2 a_{1}^{0}}, \\
& \widetilde{\beta}_{12}=\frac{a_{10}^{(1)} k_{20} g_{x}^{+}}{b_{1}^{0}}-i \frac{C_{1}^{2} \sin ^{2}\left(d_{-} \nu\right) a_{10}^{(1)} k_{20} \nu^{2} \cos ^{2}\left(d_{+} \nu\right) d_{-} d_{+}}{2 b_{1}^{0} \sqrt{d_{+}^{2}-d_{-}^{2}} \pi}
\end{aligned}
$$

Particular solution of inhomogeneous equation can be obtained using integrating:

$$
\begin{gathered}
\widetilde{v}_{30}(\xi)=-\frac{\pi^{2} a_{10}^{(1)} C_{1}^{2} \sin ^{2}\left(d_{+} \nu\right) \nu^{2} \cos ^{2}\left(d_{-} \nu\right)}{2 b_{1}^{0} d_{-}^{2}} \times \\
\left\{\begin{array}{l}
-\frac{1}{2} b_{11}^{ \pm} \rho \ln \rho \sin \theta+\frac{1}{4} b_{12}^{+} \sin 2 \theta+\sum_{j=3}^{\infty} \frac{b_{1 j}^{+}}{4(j-1)} \rho^{2-j} \sin j \theta, \quad \xi_{2}>0, \\
\frac{1}{8} b_{1}^{0} \rho^{3} \sin \theta+\frac{1}{2} b_{11}^{ \pm} \rho \ln \rho \sin \theta-\frac{1}{4} b_{12}^{-} \sin 2 \theta-\sum_{j=3}^{\infty} \frac{b_{1 j}^{-}}{4(j-1)} \rho^{2-j} \sin j \theta, \quad \xi_{2}<0 .
\end{array}\right.
\end{gathered}
$$

Finally, we obtain the full solution:

$$
\begin{gathered}
v_{30}(\xi)=\widetilde{\beta}_{11} a_{1}^{0} \rho \cos \theta-\widetilde{\beta}_{12} b_{1}^{0} \rho \sin \theta+ \\
+\frac{\pi^{2} a_{10}^{(1)} C_{1}^{2} \sin ^{2}\left(d_{+} \nu\right) \nu^{2} \cos ^{2}\left(d_{-} \nu\right)}{2 b_{1}^{0} d_{-}^{2}} \cdot\left[\frac{1}{2} b_{11}^{ \pm} \rho \ln \rho \sin \theta-\frac{1}{4} b_{12}^{+} \sin 2 \theta-\sum_{j=3}^{\infty} \frac{b_{1 j}^{+}}{4(j-1)} \rho^{2-j} \sin j \theta\right]- \\
-\sum_{j=1}^{\infty}\left(\beta_{11} a_{1 j}^{+}-\widetilde{\beta}_{11} a_{1 j}^{-}+\beta_{21} a_{2 j}^{+}\right) \rho^{-j} \cos j \theta-\sum_{j=1}^{\infty}\left(\beta_{12} b_{1 j}^{+}+\widetilde{\beta}_{12} b_{1 j}^{-}+\beta_{32} b_{3 j}^{+}\right) \rho^{-j} \sin j \theta
\end{gathered}
$$


for $\xi_{2}>0$ and

$$
\begin{gathered}
v_{30}(\xi)=\beta_{11} a_{1}^{0} \rho \cos \theta+\beta_{12} b_{1}^{0} \rho \sin \theta+\beta_{21} a_{2}^{0} \rho^{2} \cos 2 \theta+\beta_{32} b_{3}^{0} \rho^{3} \sin 3 \theta- \\
-\frac{\pi^{2} a_{10}^{(1)} C_{1}^{2} \sin ^{2}\left(d_{+} \nu\right) \nu^{2} \cos ^{2}\left(d_{-} \nu\right)}{2 b_{1}^{0} d_{-}^{2}} \cdot\left[\begin{array}{c}
\frac{1}{8} b_{1}^{0} \rho^{3} \sin \theta+\frac{1}{2} b_{11}^{ \pm} \rho \ln \rho \sin \theta- \\
1 \\
-\frac{1}{4} b_{12}^{-} \sin 2 \theta-\sum_{j=3}^{\infty} \frac{b_{1 j}^{-}}{4(j-1)} \rho^{2-j} \sin j \theta
\end{array}\right]+ \\
+\sum_{j=1}^{\infty}\left(\beta_{11} a_{1 j}^{-}-\widetilde{\beta}_{11} a_{1 j}^{+}+\beta_{21} a_{2 j}^{-}\right) \rho^{-j} \cos j \theta+\sum_{j=1}^{\infty}\left(\beta_{12} b_{1 j}^{-}+\widetilde{\beta}_{12} b_{1 j}^{+}+\beta_{32} b_{3 j}^{-}\right) \rho^{-j} \sin j \theta
\end{gathered}
$$

for $\xi_{2}<0$.

Matching terms of order $\rho^{-1} \sin \theta$ gives us:

$$
\begin{aligned}
& \frac{\alpha}{2}\left(\frac{1}{\pi} a_{10}^{(1)} k_{40}-a_{10}^{(3)} b_{10}^{(3)} k_{20}-3 a_{10}^{(3)} b_{01}^{(3)} k_{20}\right)=-\beta_{12} b_{11}^{+}-\widetilde{\beta}_{12} b_{11}^{-}-\beta_{32} b_{31}^{+}-\frac{\pi^{2} a_{10}^{(1)} C_{1}^{2} \sin ^{2}\left(d_{+} \nu\right) \nu^{2} \cos ^{2}\left(d_{-} \nu\right)}{2 b_{1}^{0} d_{-}^{2}} \cdot \frac{3 b_{13}^{-}}{8} \Leftrightarrow \\
& \frac{\alpha}{2} \cdot \frac{1}{\pi} a_{10}^{(1)} k_{40}=-\beta_{12} b_{11}^{+}-\widetilde{\beta}_{12} b_{11}^{-}-\beta_{32} b_{31}^{+}-\frac{3 b_{13}^{-} \pi^{2} a_{10}^{(1)} C_{1}^{2} \sin ^{2}\left(d_{+} \nu\right) \nu^{2} \cos ^{2}\left(d_{-} \nu\right)}{16 b_{1}^{0} d_{-}^{2}}+\frac{\alpha}{2}\left(a_{10}^{(3)} b_{10}^{(3)}+3 a_{10}^{(3)} b_{01}^{(3)}\right) k_{20} \Leftrightarrow \\
& k_{40}=\frac{2 \pi}{a_{10}^{(1)} \alpha}\left[-\beta_{12} b_{11}^{+}-\widetilde{\beta}_{12} b_{11}^{-}-\beta_{32} b_{31}^{+}-\frac{3 b_{13}^{-} \pi^{2} a_{10}^{(1)} C_{1}^{2} \sin ^{2}\left(d_{+} \nu\right) \nu^{2} \cos ^{2}\left(d_{-} \nu\right)}{16 b_{1}^{0} d_{-}^{2}}+\frac{\alpha}{2}\left(a_{10}^{(3)} b_{10}^{(3)}+3 a_{10}^{(3)} b_{01}^{(3)}\right) k_{20}\right] .
\end{aligned}
$$

Imaginary part can be obtained from summand with $\widetilde{\beta}_{12}$ :

$$
\operatorname{Im} k_{40}=\frac{-2 \pi C_{1}^{4} \sin ^{2}\left(d_{+} \nu\right) \sin ^{2}\left(d_{-} \nu\right) \nu^{4} \cos ^{2}\left(d_{+} \nu\right) \cos ^{2}\left(d_{-} \nu\right) d_{-} d_{+}\left(b_{11}^{-}\right)^{2}}{\alpha \cdot 4\left(b_{1}^{0}\right)^{2} \sqrt{d_{+}^{2}-d_{-}^{2}}} .
$$

\section{Conclusion}

The suggested procedure can be continued to obtain terms of the asymptotic expansion of any order. The results pertaining to the real part of the resonance, gives one an estimation of the shift of resonance with respect to the threshold. These results can be useful for the description of "quantum waveguide - quantum dot - quantum waveguide" systems. One can find such systems in different nanotechnological applications.

\section{Acknowledgments}

The work was partially financially supported by Russian Science Foundation grant 16-11-10330.

\section{References}

[1] Lord Rayleigh O.M. The theory of Helmholtz Resonator. Proceeding of Royal Society, 1916, 638, P. $265-275$.

[2] Morse F.M., Feshbach G. Methods of theoretical physics, V. 2. Foreign Literature Publishing House, 1960,986 p.

[3] Kiselev A.A., Pavlov B.S. The eigenfrequencies and eigenfunctions of the Laplace operator of the Neumann problem in a system of two coupled resonators. Theor. Math. Phys., 1994, 100(3), P. 354-366.

[4] Gadylshin R.R. The existence and asymptotics of poles with a small imaginary part for the Helmholtz resonator. Uspekhi of Mathematical Sciences, 1997, 52(313), P. 71-72.

[5] Borisov D.I. Discrete spectrum of an asymmetric pair of waveguides coupled through a window. Sb. Math., 2006, 197(4), P. 475-504.

[6] Achilleos V., Richoux O., et.al. Acoustic solitons in waveguides with Helmholtz resonators: Transmission line approach. Phys.Rev. E., 2015, 91, P. 023204.

[7] Martinez A., Ned' elec L. Optimal lower bound of the resonance widths for a Helmoltz tube-shaped resonator. J. Spectral Th., 2012, 2, P. 203-223.

[8] Gadyl'shin R.R. A two-dimensional analogue of the Helmholtz resonator with ideally rigid walls. Translation in Diff. Eq., 1994, 30(2), P. 201209.

[9] Gadyl'shin R.R. Influence of the position of the opening and its shape on the properties of a Helmholtz resonator. Theor. Math. Phys., 1992, 93, P. 1151-1159.

[10] Borisov D., Exner P. Distant perturbation asymptotics in window-coupled waveguides. I. The non-threshold case. J. Math. Phys., 2006, 47(10), P. 113502(1-24).

[11] Borisov D.I., Gadyl'shin R.R. On the spectrum of the Laplacian with frequently alternating boundary conditions. Theor. Math. Phys., 1999, 118(3), P. 272-277.

[12] Ilyin A.M. Matching of the asymptotic expansions of solutions. Science, 1989, 336 p.

[13] Exner P., Kreicirik D. Waveguides coupled through a semitransparent barrier: a Birman-Schwinger analysis. Rev. Math. Phys., 2001, 13, P. 307-334.

[14] Exner P., Kreicirik D. Quantum waveguides with a lateral semitransparent barrier: spectral and scattering properties. J. Phys. A, 1999, 32, P. 4475. 
[15] Vorobiev A.M., Bagmutov A.S., Popov A.I. On formal asymptotic expansion of resonance for quantum waveguide with perforated semitransparent barrier. Nanosystems: Physics, Chemistry, Mathematics, 2019, 10(4), P. 415-419.

[16] Behrndt J., Langer M., Lotoreichik V. Boundary triples for Schrodinger operators with singular interactions on hypersurfaces. Nanosystems: Phys. Chem. Math., 2016, 7(2), P. 290-302.

[17] Mantile A., Posilicano A. Laplacians with singular perturbations supported on hypersurfaces. Nanosystems: Phys. Chem. Math., 2016, 7(2), P. 315-323.

[18] Exner P., Kondej S., Lotoreichik V. Asymptotics of the bound state induced by delta-interaction supported on a weakly deformed plane. $J$. Math. Phys., 2018, 59, P. 013051.

[19] Behrndt J., Exner P., et.al. Approximation of Schroedinger operators with delta-interactions supported on hypersurfaces. Math. Nachr., 2017, 290, P. 12151248.

[20] Popov I.Yu. The operator extension theory, semitransparent surface and short range potential. Math. Proc. Cambridge Phil. Soc., 1995, 118, P. 555-563.

[21] Popov I.Yu. The extension theory, domains with semitransparent surface and the model of quantum dot. Proc. Royal Soc. London A, 1996, 452(1950), P. 1505-1515.

[22] Popov I.Yu. The extension theory and the opening in semitransparent surface. J. Math. Phys., 1992, 33(4), P. 1685-1689.

[23] Tikhonov A.N., Samarskii A.A. Equations of Mathematical Physics., M.:Science, 1972, 531 p. 